\title{
A Qualitative Cross-Cultural Comparison of Well-Being Constructs: the Meaning of Happiness, Life Satisfaction, and Social Support for German and Chinese Students
}

\author{
Julia Brailovskaia ${ }^{1} \cdot$ Muyu Lin ${ }^{1,2}$ (D) Saskia Scholten ${ }^{3} \cdot$ Meixia Zhu ${ }^{4,5} \cdot$ Yue Fu ${ }^{4,6}$. \\ Meihua Shao ${ }^{7,8}$. Shuqing $\mathrm{Hu}^{7,9} \cdot$ Xuan $\mathrm{Li}^{7}$. Wenting Guo ${ }^{7}$ - Dan $\mathrm{Cai}^{4}$. Shan $\mathrm{Lu}^{7}$. \\ Jürgen Margraf ${ }^{1}$
}

Accepted: 3 September 2021 / Published online: 5 October 2021

(c) The Author(s) 2021

Keywords Subjective happiness · Life satisfaction · Perceived social support · Qualitative analysis $\cdot$ Measurement invariance $\cdot$ Focus groups $\cdot$ Germany $\cdot$ China

\section{Introduction}

As early as 1948, the importance and distinctions between social and mental well-being have been clearly established by the constitution of the World Health Organization; "health is a state of complete physical, mental, and social well-being" (World Health Organization, 1948). This statement was written to be applicable worldwide. Subjective well-being - an important indicator of mental wellness - refers to the evaluation of one's own declared quality of life, including how one feels about life and how well one sees him-/herself functioning in life (Keyes, 2012, p. 5). The literature on subjective well-being mainly includes happiness, life satisfaction, and positive affect (Diener, 2009, p. 11). Meanwhile, the

Muyu Lin and Julia Brailovskaia share the co-first authorship of this article.

Muyu Lin

muyu.lin@rub.de

1 Department of Clinical Psychology and Psychotherapy, Mental Health Research and Treatment Center, Ruhr-Universität Bochum, Massenbergstr 9-13, 44787 Bochum, Germany

2 Clinical Psychology of Social Interaction, Institute of Psychology, Berlin School of Mind and Brain, Humboldt-Universität Zu Berlin, Berlin, Germany

3 Universität Koblenz-Landau, Pain and Psychotherapy Research Lab, Ostbahnstr. 10, 76829 Landau in der Pfalz, Germany

4 Department of Psychology, Shanghai Normal University, Shanghai, China

5 Department of Education, Qingdao Binhai University, Qingdao, China

6 Shanghai Pudong Mofan Experimental Junior High School, Shanghai, China

7 Beijing Key Laboratory of Learning and Cognition and Department of Psychology, School of Psychology, Capital Normal University, Beijing, China

$8 \quad$ Yichun Early Childhood Teachers College, Yichun, China

9 Xiang Xi Ya Si Experimental Middle School, Jishou, China 
construct of social well-being is less familiar to many people. McDowell, (2006, p. 152) defined social well-being as an aspect of health that concerns how one gets along with others, interacts with social institutions, observes societal mores, and how others react. Studies on social well-being mostly focus on social support (e.g., quality or number of social contacts) and social adjustment (e.g., satisfaction with relationships or performance in social roles). This disentanglement of both dimensions of well-being (i.e., subjective well-being and social well-being) has been supported empirically across different cultures (Joshanloo et al., 2014, 2016; Joshanloo, 2019a, 2019b; Ryan \& Deci, 2001; Teismann \& Brailovskaia, 2019). Based on this framework, the current study focused on the following representations of the two dimensions of well-being: happiness and satisfaction with life as a measure of subjective well-being, and perceived social support as a measure of social well-being.

\subsection{Happiness}

A general definition of happiness is "an internal experience of a positive state of mind" (Lu \& Shih, 1997, p. 182). The meaning of happiness can differ across cultures and even across individuals. While the Western conceptualization of happiness stresses an intrapersonal focus, internal evaluation and contentment, its hedonistic pursuit is regarded as unworthy and shameful in Chinese culture (Lu \& Shih, 1997). As a consequence, European and north-American cultures focus on pleasantness. In contrast, East-Asian cultural groups are aware of the arousal dimension that implies keeping a good balance of mind or staying calm and content (Kitayama \& Markus, 2000).

\subsection{Satisfaction with Life}

Shin and Johnson, (1978, p. 478) define life satisfaction as "a global assessment of a person's quality of life according to his chosen criteria". Diener et al., (1985) further added that "judgments of satisfaction are dependent upon a comparison of one's circumstances with what is thought to be an appropriate standard. It is important to point out that the judgment of how satisfied people are with their present state of affairs is based on a comparison with a standard, which each individual sets for him- or herself'. Consistently, people in collectivistic cultures often report lower life satisfaction than people in individualistic cultures (Bieda et al., 2017; Kang et al., 2003; Tov \& Au, 2013). Moreover, in societies with high levels of economic and physical security that typically foster a climate of trust and self-expression, positive emotions are more strongly related to life satisfaction than in nations in which individuals focus on their daily survival (Kuppens et al., 2008).

\subsection{Perceived Social Support}

Perceived social support can be defined as "the result of cognitive-emotional information processing and the evaluation of present and past social interactions. Persons experienced or expected support (assistance) to master tasks, cope with stress and reach personal goals in those interactions. Hence, social support is primarily a perceived or anticipated support from the social network." (Fydrich et al., 2009, p. 43). It could be hypothesized that Western cultures emphasize informational and instrumental aspects of social support because 
the individual is viewed as independent from others; hence, helping might be conceptualized as a mutual service. In contrast, as Asians view a person as fundamentally connected with others and responsible for maintaining social harmony, expressing personal problems or requesting help from others might not be a desirable social behavior (Taylor et al., 2004).

\subsection{Cross-cultural comparisons on well-being}

Cross-cultural research on well-being has revealed multi-level differences, from the denotation and connotation of the construct to its experiences and moderators (e.g., Bieda et al., 2017; Joshanloo, 2014; Kitayama \& Markus, 2000; Taylor et al., 2004). Eastern and Western cultures differ from each other in many aspects, such as in collectivism vs. individualism (Markus \& Kitayama, 1991), which may influence well-being on various levels. On a conceptional level, role obligation and dialectical balance were emphasized for the social well-being of Chinese students; for students with a European and American background, individual well-being encompassed explicit pursuit and personal accountability (Lu \& Gilmour, 2004). On a response bias level, people from a traditional Confucian society tend to suppress the expression of positive affect, while American culture typically emphasizes the expression of positive intrapersonal feelings (Lau et al., 2005; Ying, 1989). Such bias may contribute to a higher rating of subjective well-being in Western countries (i.e., North America, Western Europe, and Australia) than in Eastern countries (i.e., China, Korea, and Japan) (e.g., Helliwell, Huang, \& Wang, 2017; OECD, 2021). Against this background, the current study investigated Eastern and Western cultural differences in happiness, satisfaction with life, and perceived social support between China and Germany.

Many of the available cross-cultural comparisons are based on observed scores on multi-item scales. However, the validity of such scales depends on the assumption that people from different cultural groups respond to the items in the same way. This assumption can be statistically tested via measurement invariance. Bieda et al., (2017) and Lin et al., (2019) conducted large-scale studies with university students from China and Germany to test cross-cultural measurement invariance of scales assessing positive well-being. Strong cross-cultural measurement invariance (i.e., equal intercepts across cultures) was established for the scale assessing general positive mental health (Bieda et al., 2017). Meanwhile, only weak invariance (i.e., equal loadings on the latent construct across groups) or partial strong invariance (i.e., equal item intercept across groups) was established across Germany and China concerning the Subjective Happiness Scale (SHS; Lyubomirsky \& Lepper, 1999), the Satisfaction with Life Scale (SWLS; Diener et al., 1985), and the Social Support Questionnaire (F-SozU K-14; Fydrich et al., 2009). Given that there are scales that can reach strong cross-cultural invariance (e.g., the Positive Mental Health-Scale across China, Germany, and Russia in Bieda et al., (2017); the Depression, Anxiety, and Stress Scales across Poland, Russia, the United Kingdom, and the United States of America in Scholten et al., (2017)), it is essential to clarify why the scales that assess happiness, life satisfaction, and social support did not show this form of invariance and to explore the possible source of biases that might explain the non-invariances.

If scale invariance is not achievable, multiple potential reasons need to be considered: (1) the meaning of a concept across cultural groups (i.e., the content of a specific item fits better in one group than in another group, or the overall understanding of the concept differs across groups), (2) poor translation, (3) the effect of social desirability on response style, (4) differences in the value given to certain aspects, or (5) different reference frameworks (Chen, 2008). These reasons might at least partly explain why Western-developed. 
Instruments do not always appropriately transfer to research in non-Western countries. From a statistical point of view, there have been multiple propositions on how to deal with non-invariance (e.g., Byrne et al., 1989; Chen, 2008; Steenkamp \& Baumgartner, 1998; Vandenberg \& Lance, 2000). However, linking qualitative research methods to these elaborate quantitative approaches could play a role in uncovering the reasons for non-invariance by exploring the differences in the meaning of well-being across cultures (Hines, 1993; Scholten et al., 2017).

\subsection{Qualitative Study as Follow-up Explanation for the Non-invariance}

The aim of the present study was to explore cross-cultural differences in the concepts and questionnaires of social support, life satisfaction, and happiness, which have statistically been identified in terms of non-invariance by Bieda et al., (2017) and Lin et al., (2019) across Chinese and German participants. Considering the lack of an established way to explore such biases, we proposed adding a qualitative approach based on focus groups (Krueger \& Casey, 2015). Specifically, we aimed to explore whether there are cross-cultural differences in the definitions of the three constructs, and whether the non-invariant items were due to translation error, differences in understanding, difficulty level, response style, and/or other sources of bias. To the best of our knowledge, this is the first qualitative, in-depth study undertaken to understand findings of measurement non-invariance. a strength of the study is that we also seek to understand the cross-cultural differences from a contextual point of view. As a follow-up explanatory study (Creswell \& Plano Clarke, 2017) of the quantitative results presented in Bieda et al. (2017) and Lin et al. (2019), present findings may contribute to the understanding of reported non-invariance and complement the preceding quantitative results. Moreover, they can reveal potential ways, in which available instruments that assess the investigated constructs might be modified, or which factors should be considered when developing new instruments to enable measurement invariance in cross-cultural studies.

\section{Materials and Methods}

\subsection{Participants}

The present study is part of the ongoing BOOM (Bochum Optimism and Mental Health) research program investigating the risk and protective factors of mental health in different countries (see boom.rub.de). The responsible Ethics Committee approved the study implementation. All participants gave written consent before entering a focus group. Nine groups (five in Germany and four in China) were included.

\subsubsection{German Sample}

In November 2016, an e-mail with a participation invitation for the focus groups was sent to a random sample of 200 persons from the BOOM participant pool in Bochum, Germany. The inclusion criterion for participation was sufficient knowledge of the German language. Overall, 33 persons ( $81.8 \%$ female; age (years): $23.03 \pm 5.97$, range: $18-41$; occupation: $87.9 \%$ students, $12.1 \%$ employees; marital status: $57.6 \%$ single, $42.4 \%$ in romantic relationship) participated in one of the five focus groups. 


\subsubsection{Chinese sample}

In January 2017, invitations were sent to 50 Beijing BOOM participants who left contact information, while online advertisements were sent to Shanghai BOOM participants who studied or worked in Shanghai. In total, 28 individuals (85.2\% female; age (years): $22.56 \pm 1.05$, range: $21-25$; occupation: $63 \%$ students, $33.3 \%$ employees, $3.7 \%$ trainees; marital status: $59.3 \%$ single, $37 \%$ in romantic relationship, $3.7 \%$ missing) took part in one of the four focus groups (two in Beijing, two in Shanghai).

\subsection{Scales and items discussed in the focus groups}

The following scales and measurement inequivalent items were presented to the participants during the focus group session. The Chinese and German versions of the non-invariant items are presented in Table S1 (supplementary materials).

\subsubsection{Social Support Questionnaire (F-SozU K-14)}

The unidimensional questionnaire F-SozU K-14 (Fydrich et al., 2009) assesses perceived social support with 14 items that are rated on a 5-point Likert-type scale from 1 (not true at all) to 5 (very true). Cronbach's alpha was .95 in both German and Chinese samples (Bieda et al. 2017). In Bieda et al. (2017) and Lin et al. (2019), the intercepts of Item 3 (I experience a lot of understanding and security from others.), Item 5 (If necessary, I can easily borrow something I might need from neighbors or friends.), Item 8 (I have friends and family who will simply just hug me.) and Item 11 (There are people who share joy and sorrow with me.) had to be released in order to establish measurement invariance of the F-SozU K-14.

\subsubsection{Satisfaction with Life Scale (SWLS)}

The SWLS (Diener et al., 1985) measures global life satisfaction. It consists of five items rated on a 7-point Likert-type scale $(1=$ strongly disagree; $7=$ strongly agree $)$. Cronbach's alpha was .89 in the German and .88 in the Chinese sample (Bieda et al., 2017). The factor loading of Item 2 (The conditions of my life are excellent.) and the intercepts of Item 1 (In most ways my life is close to my ideal.) and Item 3 (I am satisfied with my life.) could not be constrained when testing measurement invariance across countries (Bieda et al., 2017).

\subsubsection{Subjective Happiness Scale (SHS)}

The SHS (Lyubomirsky \& Lepper, 1999) assesses overall subjective happiness with four items rated on a 7-point Likert-type scale. Cronbach's alpha was .87 in the German and .74 in the Chinese sample (Bieda et al., 2017). A measurement invariance test of the SHS indicated that Item 3 (Some people are generally happy. They enjoy life regardless of what is going on, getting the most out of everything.) and Item 4 (Some people are generally not very happy. Although they are not depressed, they never seem as happy as they might be.) did not measure the same factor equivalent across groups (loading and intercept variance) (Bieda et al., 2017). 


\subsection{Procedure}

First, an English version of a focus group route was developed by a team of German and Chinese psychologists. The questioning route was translated into German and Mandarin using the translation-back-translation-modification procedure (Berry, 1989) to enable standardized implementation of the focus groups in both countries. Next, a pilot focus group was assembled in Germany to test the procedure. As no further modifications of the procedure were deemed necessary, the results of the pilot group were also included in all analyses. Then, eight respective focus groups were conducted in Germany (four groups) and in China (four groups). In each group, only two of the three constructs were discussed: "perceived social support" was discussed in all groups, "subjective happiness" in three German groups and in two Chinese groups, and "life satisfaction" in another two German groups and two Chinese groups. This reduced the probability of attentional overload for participants being asked to maintain a high level of attention and concentration over a longer period of time. For each construct, the definition was discussed first. Afterward, the non-invariant items related to the construct were discussed. Participants rated each item ("fit", "not fit", or "not sure") and explained whether it measured the definition discussed before; then, they described their free associations related to the item. Notably, the focus group sessions lasted approximately $2 \mathrm{~h}$ with a 15 min break after discussing the first construct. Discussing all three constructs following these three steps would have extended the duration of the sessions up to $3 \mathrm{~h}$ and increased the risk of participants scrambling the constructs and discussion points due to decreasing levels of attention and concentration. The detailed procedure of one focus group session is provided in the supplementary material. All focus groups were audio-recorded. To preserve anonymity, participants were addressed with nicknames during the focus group sessions. Participants were compensated with course credits or cash (15 Euro in Germany, 100 RMB in China).

\subsection{Qualitative and Quantitative Analyses}

All raters were trained psychologists and native speakers of the particular focus group language. The qualitative analyses of the construct definitions and the free association/ reasons for fit/not-fit of each item followed the steps of the inductive qualitative content analysis presented by Mayring (2015). First, the audio recordings of all group discussions were transcribed word-for -word. Second, the statements/phrases that were meaningful for the analyses were identified, highlighted, and copied into a separate document (in contrast, the moderators' comments and participants' acknowledgment of the moderators' clarifications were considered irrelevant for the analyses, and thus, they were excluded). Third, the content of each statement was summarized (e.g., statement: "The first thing that comes to mind when I hear "perceived social support" is someone giving me some money to help me pay for my flat, which would solve a lot of my problems. I have to work hard to get this money. So, I need this money."; summary: someone gives some money to help to pay for flat, solves problems). Fourth, for each summarized statement, a preliminary category was suggested (e.g., providing material support). Fifth, similar categories were assigned to superordinate categories (e.g., material support). For each superordinate category, a definition was formulated (e.g., providing of financial resources or objects, different from emotional support), and a typical example was given (e.g., money to pay for float). The third to fifth steps were conducted separately by two raters. Sixth, the two raters discussed all 
categories and developed a final category system together (in Germany) or in collaboration with a third rater (in China).

In the final step, the raters calculated how frequently each category was mentioned (an example of the entire procedure is listed in Table S2 in the supplementary material).

In both countries, there were two rounds for all three constructs. A percent agreement calculation was chosen to quantify the interrater agreement (i.e., the proportion of the same category from all summarized statements). In the first round, the interrater agreement was $85 \%$ in Germany and $71 \%$ in China for "subjective happiness", $90 \%$ and $61 \%$, respectively, for "life satisfaction", and $87 \%$ and $61 \%$ for "perceived social support". In the second round, an interrater agreement of $100 \%$ was reached for all constructsin both countries.

For the rating of item fit, percentages of fit/not-fit/not-sure were calculated for each country. Chi-square tests were applied to examine whether the frequency of voting for fit/ not fit/not sure differed between the two countries.

\section{Results and Discussion}

The final category systems for the definition of all constructs-including the frequency of each category-are presented in Table 1 (subjective happiness), Table 2 (life satisfaction), and Table 3 (perceived social support). Categories that were mentioned only once (see Table S3, supplementary material) were considered single opinions and were omitted from tables and discussion.

\subsection{Definition of Happiness}

\subsubsection{Results}

As summarized in Table 1, in both countries, participants mentioned quality of life, positive emotions, and social contacts as aspects of happiness; these results are in line with Keyes' model (2007). Social contact was the least frequently mentioned aspect among the three in Germany (e.g., "Happiness is spending my free time with my best friends."), but the most frequent one in China (e.g., "Happiness to me means someone at home cares for me, e.g., asking when I will be home for dinner." $)$.

\subsubsection{Discussion}

German participants differentiated between two types of happiness: (1) Uncontrollable, i.e., good luck; (2) Controllable, i.e., one's ability to achieve something. They emphasized the subjectivity of the definition of happiness and its natural brevity. Meanwhile, Chinese groups viewed happiness from a different angle: it could appear as a result of a goal/dream being fulfilled (e.g., "The things I look forward to come true.") or while pursuing the goal/ dream (e.g., "When I am striving to achieve my goal").

${ }_{1}^{1}$ All examples/quotations listed in the results section were originally in Chinese or in German. 


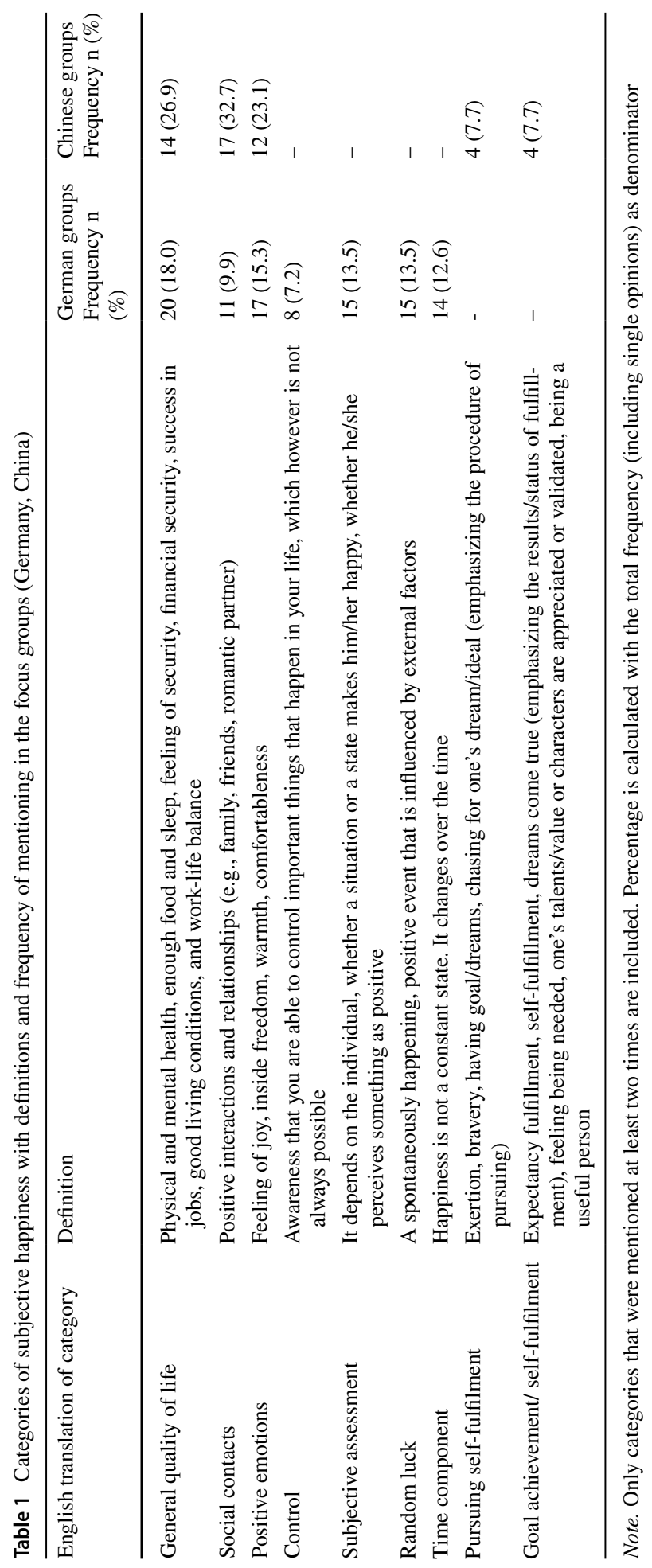




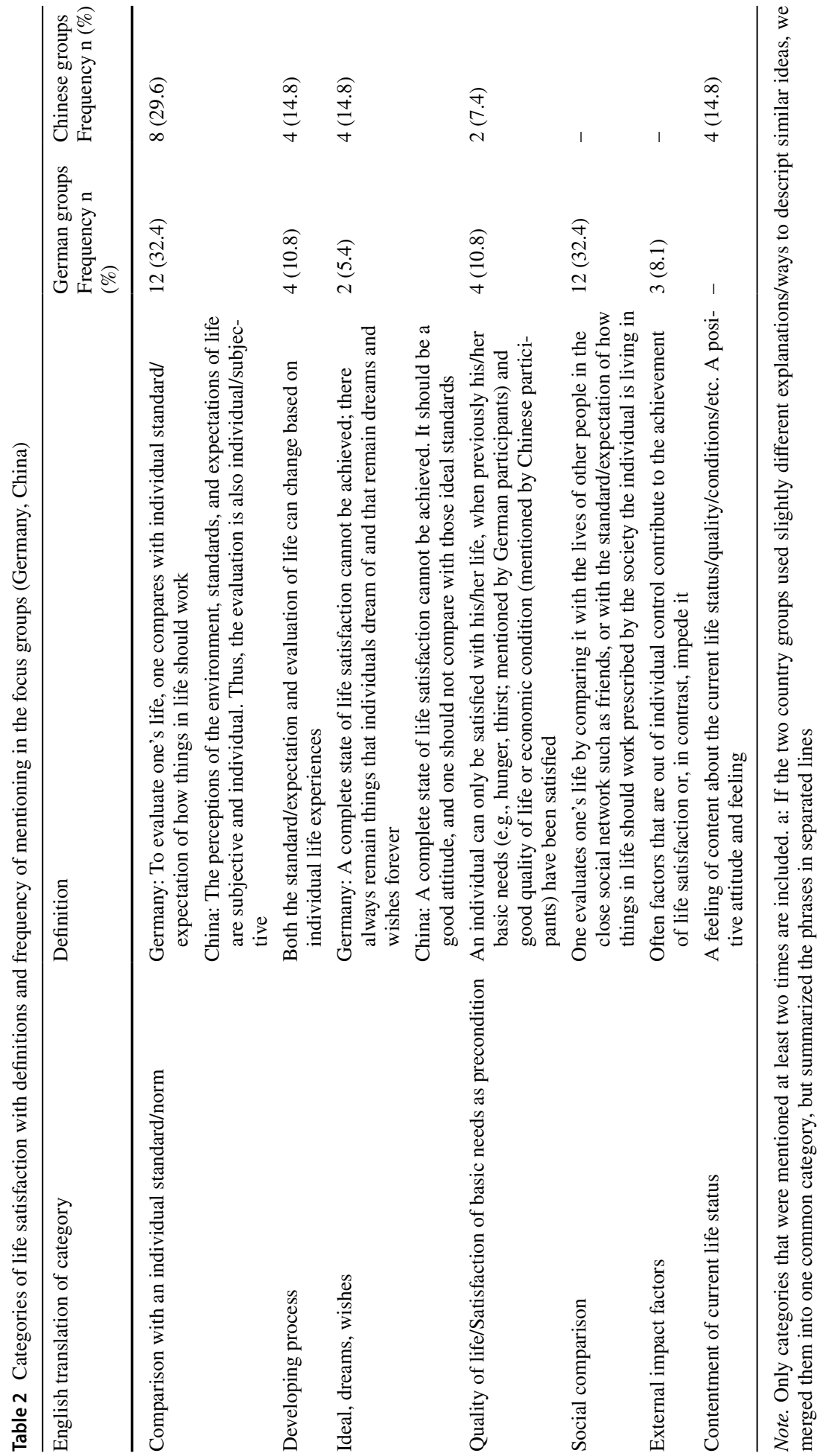




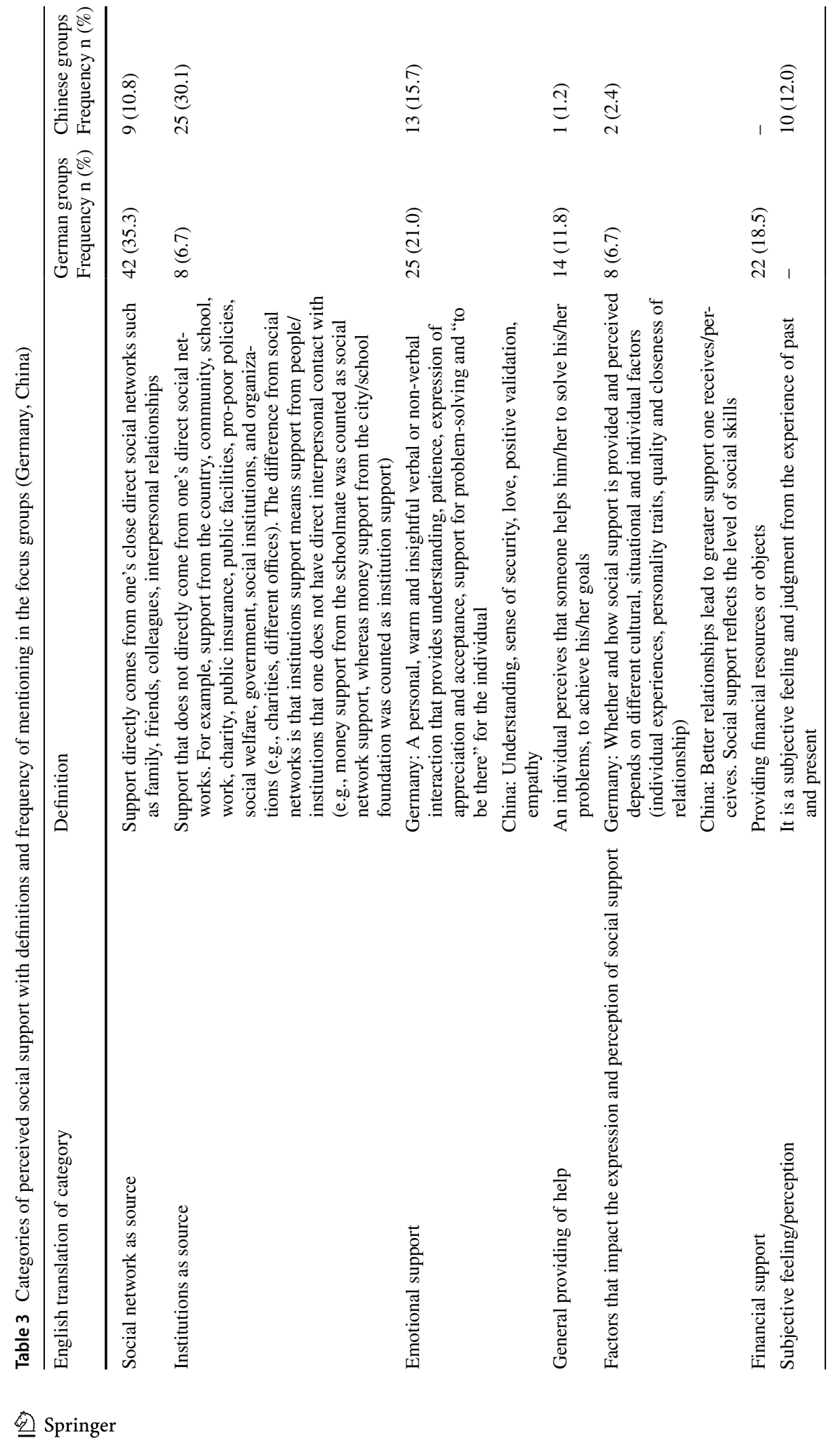




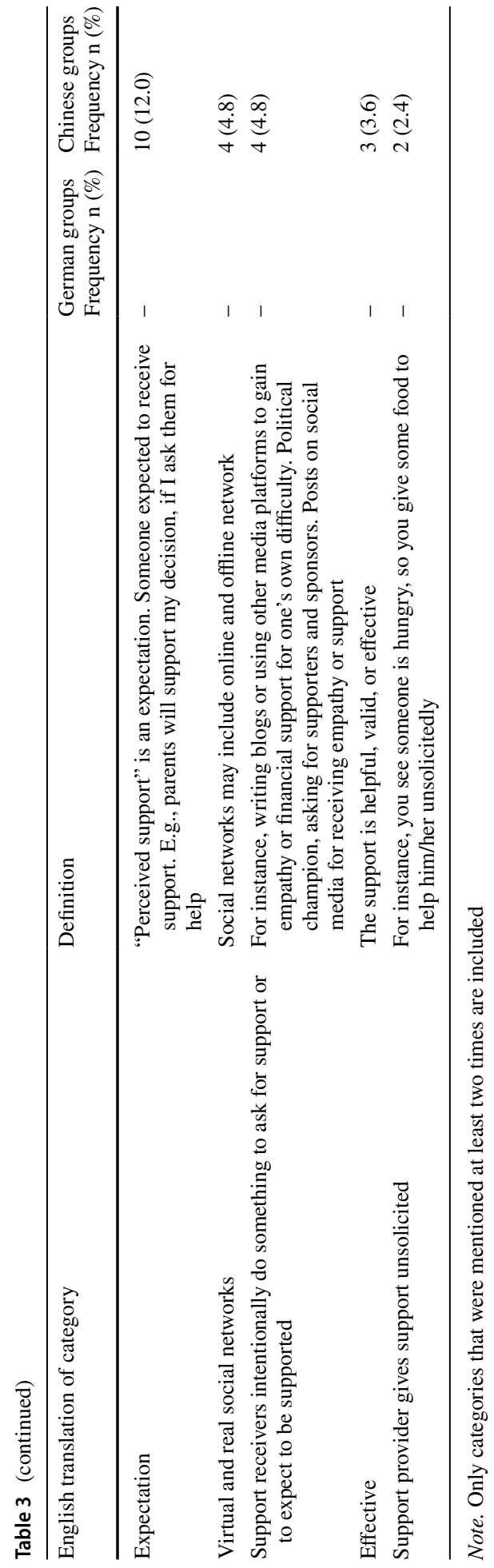




\subsection{Definition of Life Satisfaction}

\subsubsection{Results}

In both countries, participants mentioned an individual realistic standard and an ideal standard of life satisfaction that can change over time, as well as the perception of current satisfaction (see Table 2). Although participants from both countries mentioned quality of life as an important facet of life satisfaction, German students focused more on basic needs fulfillment (e.g., enough food, a place to live), while Chinese students referred to good living conditions (e.g., good economic conditions, high salary/income).

\subsubsection{Discussion}

The variation in results may be due to the different economic development levels in the two countries (The World Bank, 2020). The Chinese groups mentioned a positive attitude toward life and contentment with their current situation as part of life satisfaction, while the German groups mentioned that satisfaction may come from comparing their situation with a social norm/social standard or with an individual standard.

\subsection{Definition of Perceived Social Support}

\subsubsection{Results}

Participants from both countries frequently mentioned their sources of support (i.e., private networks, such as friends and family, and society as a whole). In the German groups, direct social network support was mentioned most frequently, while Chinese students gave many examples of support from "the society". They emphasized donations/help/support from the community (e.g., companies, charities, and government policies that provide benefits for young students from poor families), i.e., support from people/entities who/that do not know you directly. However, financial support and material support were more frequently mentioned in the German groups than in the Chinese groups. In addition, only the Chinese groups gave examples of expectations of unsolicited support and implicit/indirect ways of "asking for help" (e.g., posting one's problem on social media, expecting to gain empathy). Furthermore, participants in both countries mentioned emotional support and a general term of help.

\subsubsection{Discussion}

The emphasis on different sources of support between countries (the direct social network in Germany vs. "the society" in China) could be partly due to a translation bias. Social support is usually translated into “社会支持shènùi zhīchí” (see Table S1) in Chinese, which can be interpreted literally as "support from the big society/government/community" by individuals not familiar with this psychological term. Overall, the perceived level of social support may depend on different cultural, situational, and individual factors, such as whether one has good interpersonal relationships. 


\subsection{The evaluation of item fit and free associations}

The results of the evaluation of item fit and the reasons for "not fit" or "not sure" ratings are summarized in Table 4. The participants from the two countries were similar in their evaluation pattern on most of the items, except for Item 4 of SHS $\left(\chi^{2}=9.771, p=0.008\right.$, Cramer's $\mathrm{V}=0.536)$, as well as Item $3\left(\chi^{2}=6.209, p=0.045\right.$, Cramer's $\left.\mathrm{V}=0.322\right)$, Item 5 $\left(\chi^{2}=18.709, p<0.001\right.$, Cramer's $\left.\mathrm{V}=0.742\right)$ and Item $8\left(\chi^{2}=8.996, p=0.011\right.$, Cramer's $\mathrm{V}=0.387$ ) of F-SozU K-14. A detailed discussion of the item fit and the reasons for unfit/ unsure selections (see Table 4) and free associations (see Table S4-S6) provided by the students are presented for each targeted item.

\subsubsection{Item 3 of SHS (Some people are generally happy. They enjoy life regardless of what is going on, getting the most out of everything.)}

3.4.1.1 Results In both countries, the evaluation of Item 3 of SHS was relatively low (i.e., only $50-56 \%$ of the participants thought this item measures the constructs). Reasons for unfit or doubt were that this item seems to measure optimism rather than happiness (see Table 4).

\subsubsection{Discussion}

A source of bias for this item may come from the translation (see Table S1). Its Chinese wording can be understood as "try my best no matter what to enjoy life" or "be the best person no matter what". The German wording (sie machen aus allem das Beste, i.e., they make the best of everything) is closer to the English version. Chinese groups argued that high ambition causes a desire to achieve the best, but that this is not comparable to happiness. The German groups emphasized that doing the best is a characteristic of optimism rather than happiness. These different points of view may explain the variant loadings of this item in Bieda et al. (2017).

\subsubsection{Item 4 of SHS (Some people are generally not very happy. Although they are not depressed, they never seem as happy as they might be.)}

\subsubsection{Results}

Regarding Item 4 of SHS, a large percentage of the Chinese students (56.3\%) agreed that the item measures happiness, while most German students $(66.7 \%)$ thought this item does not. The reasons for unfit provided by both groups were similar, including that the expression may not reveal the true mood/emotion being felt. Some German students mentioned that unhappiness might be reflected by negative feelings, while a Chinese group associated this item with the absence of happiness, e.g., someone who is hard to cheer up, which does not necessarily equate to depression (see Table S4). 


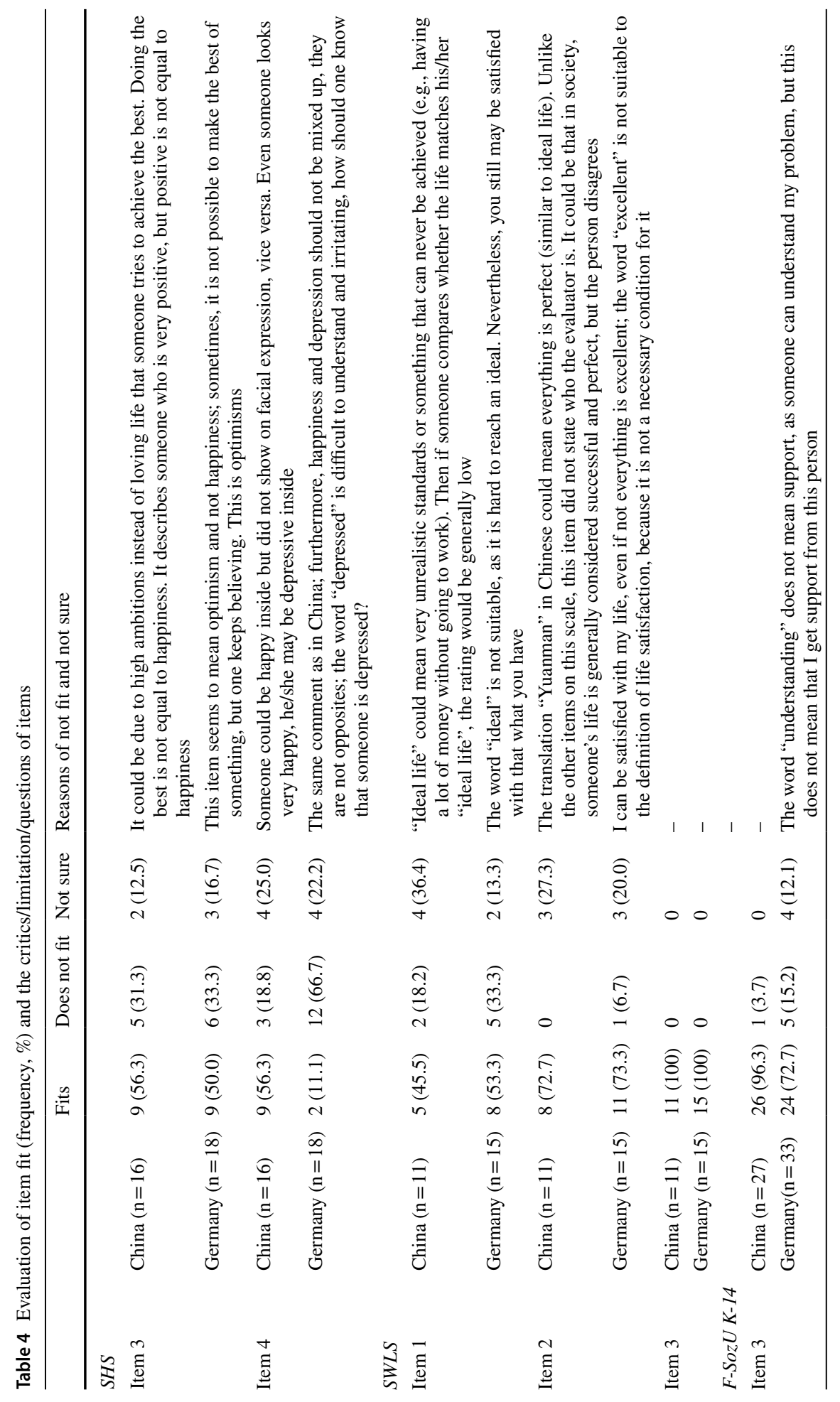




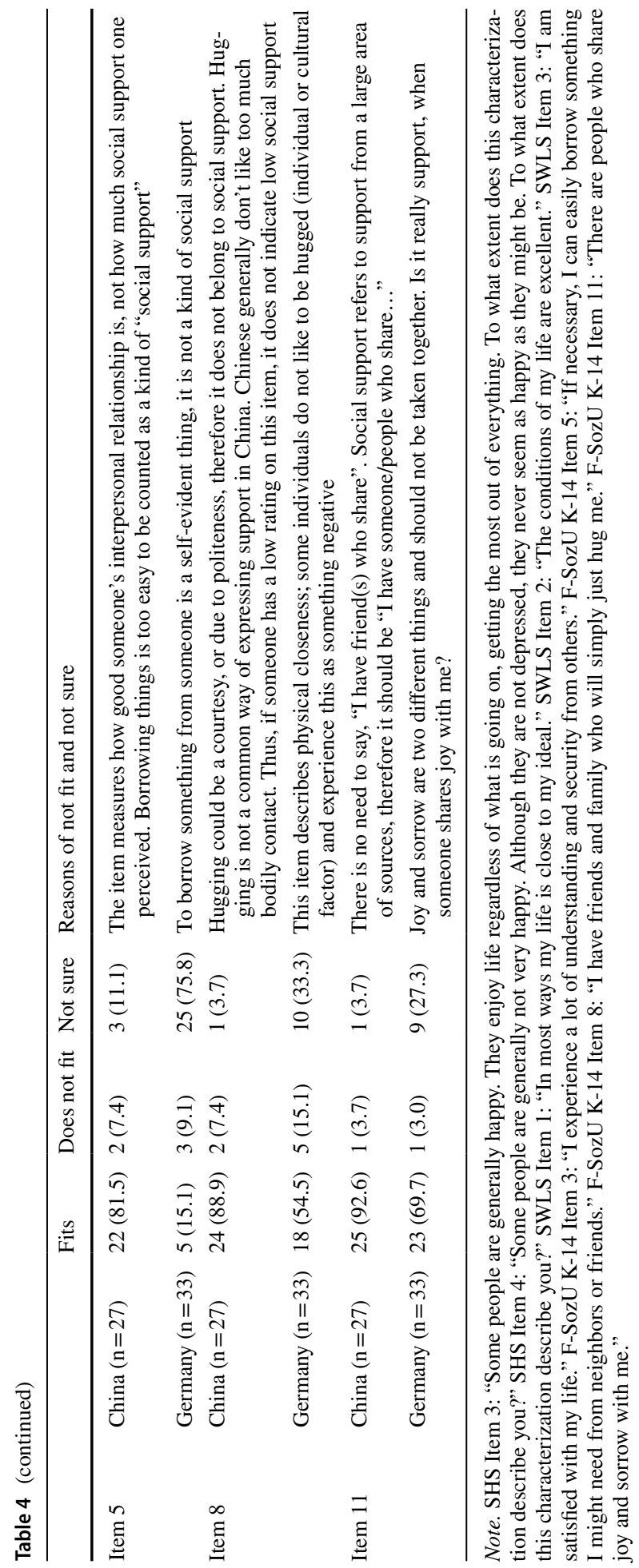




\subsubsection{Discussion}

The different association or understanding of the items may indicate a source of bias, i.e., German participants may rate the item using depression as a framework. In contrast, Chinese participants may mainly focus on the lack of happiness.

\subsubsection{Item 1 of SWLS (In most ways my life is close to my ideal.)}

\subsubsection{Results}

Even when disregarding country differences, the evaluation of Item 1 of SWLS was relatively low (i.e., only $45-53 \%$ of the participants thought these items measure the constructs). Reasons for unfit or doubt were that this item included the word "ideal"which is not suitable and unachievable (see Table 4) —and that life satisfaction may relate more to a "good-enough-standard" (e.g., the "basic needs" mentioned in the German groups and the "good living conditions" mentioned in the Chinese groups, see Table 2).

\subsubsection{Discussion}

Regarding possible source of bias, both country groups mentioned that the word "ideal" sets an extremely high standard for the ratings (see Table 4 and Table S4). The German students thought that "Idealvorstellung (ideal)" is hard to reach, but one may still be satisfied with what they have. On an extremer note, the Chinese students mentioned that “理想lixiăng (ideal)" is unrealistic and never achievable. If someone compares their current life to an "ideal" standard, the rating would be generally low. This could be one reason for the Chinese group rating this item lower than the German group in Bieda et al. (2017).

\subsubsection{Item 2 of SWLS (The conditions of my life are excellent.)}

\subsubsection{Results}

As described in Table 4, both country groups agreed that Item 2 of SWLS measures life satisfaction.

\subsubsection{Discussion}

A translation bias could lead to different rating standards. In the Chinese version, the word “圆满yuánmăn" is used, which means "perfect and complete" in the German version, the word used is "ausgezeichnet"-which means "outstanding" — and is not as extreme as "perfect and complete". The German focus groups emphasized that the word "excellent" is not a suitable condition for evaluating life satisfaction. For instance, one can be satisfied with life, without everything being excellent. This different understanding may explain why in Bieda et al. (2017), this item was more closely related to life 
satisfaction in the Chinese sample than in the German sample $(\lambda=0.904$ compared to $\lambda=0.667)$.

\subsubsection{Item 3 of SWLS (I am satisfied with my life.)}

\subsubsection{Results}

Although both groups agreed that Item 3 of SWLS measures life satisfaction, interestingly, their associations differed (see Table S5). The Chinese focus groups mentioned detailed aspects of satisfaction (basic needs are fulfilled, great quality of life, etc.), while the German focus groups often mentioned that this item referred to individual, subjective standards.

\subsubsection{Discussion}

This could be the reason why, in Bieda et al. (2017), the German students were more likely to endorse this item than the Chinese students.

\subsubsection{Item 3 of F-SozU K-14 (I experience a lot of understanding and security from others.)}

\subsubsection{Results}

Most of the Chinese students (96.3\%) agreed that Item 3 of F-SozU K-14 measures perceived social support; the proportion was lower in the German sample (75.8\%).

\subsubsection{Discussion}

The main reason for hesitation mentioned in the German groups was that the word "Verstaendnis" (understanding) does not automatically imply support (see Table 4), given that understanding something does not necessarily mean that someone will provide support.

\subsubsection{Item 5 of F-SozU K-14 (If necessary, I can easily borrow something I might need from neighbors or friends.)}

\subsubsection{Results}

Most Chinese students (81.5\%) agreed that Item 5 of F-SozU K-14 measures perceived social support. The majority of the German students (72.7\%) did not agree with it. They questioned whether borrowing something is a form of social support (see Table 4).

\subsubsection{Discussion}

Regarding the free association of this item (see Table S7), both country groups mentioned that the drive to borrow things depends on one's self-confidence (or courage) 
and relationships with others, and that borrowing things would improve interpersonal relationships. The Chinese students also pointed out that they usually prefer to buy instead of borrow items. The Mandarin words for "borrow” are “麻烦别人máfán biérén (trouble/inconvenience/burden others)" and “欠人情qiàn rénxíng (owe someone a favor)", which creates a social conflict that is generally frowned upon in East-Asian culture. This issue might at least partly explain why the Chinese sample was less likely to endorse this item than the German sample in Bieda et al. (2017).

\subsubsection{Item 8 of F-SozU K-14 (I have friends and family who will simply just hug me.)}

\subsubsection{Results}

Most of the Chinese students (88.9\%) agreed that Item 8 of F-SozU K-14 measures perceived social support, compared to about half of the German students (54.5\%). Some German students believed that this item describes physical closeness and that some individuals may not like to be hugged; therefore, this item may not measure social support (see Table 4).

\subsubsection{Discussion}

This item may also reflect a difference in customs or culture. The Chinese groups mentioned that, although many young Chinese individuals are more open to hugging than their parent generations (due to westernization in metropolitan areas), frequent body contact is still not the top welcome gesture. They would hug their lovers, children, or very close friends, but not family members, friends, or the opposite gender. It is not odd that the German sample was more likely to endorse this item than the Chinese sample (Bieda et al., 2017; Lin et al., 2019), given that hugging is a more frequent part of German behavioral repertoire.

\subsubsection{Item 11 of F-SozU K-14 (There are people who share joy and sorrow with me.)}

\subsubsection{Results}

Both country groups agreed that Item 11 of F-SozU K-14 measures social support (see Table 4).

\subsubsection{Discussion}

As presented in Table S7, the source ofnon-invariance of this item may be due to translation bias and cultural differences. In the Chinese version, "there are people who..." was translated to "I have friends who...", which may narrow the range of references, leading to a smaller possibility of endorsing the item in this sample. For example, five members from the Chinese group mentioned that they only share sorrow with strangers online, because sharing it with people they care about could be a burden. Furthermore, in Chinese culture, "don't burden someone" can be a way of being kind or caring for 
loved ones. Such cultural differences may also lower the possibility of endorsement of this item in the Chinese sample (Bieda et al., 2017; Lin et al., 2019).

\section{General Discussion and Conclusions}

For a long time, the use of the same instruments after a translation-back translation procedure (Berry et al., 1989) was recognized as a functional and common way to investigate cross-cultural differences in well-being. However, research that focused on the structure and consistency of such instruments described statistical discrepancies that impede their cross-cultural measurement invariance and the interpretation of appropriate findings (e.g., Bieda et al., 2017; Lin et al., 2019). The reasons for non-invariance remained unclear. In the present study, the meaning of and reasons for non-invariance of happiness, life satisfaction, and perceived social support - constructs that represent different dimensions of well-being - were investigated (e.g., Diener, 1984; Joshanloo et al., 2016; Ryff, 1989). We conducted nine focus groups with students (five in Germany and four in China) to discuss their understanding of the concepts and questionnaires (Krueger \& Casey, 2015), and analyzed the results with a qualitative content analysis (Mayring, 2015; Schreier, 2012). The current findings partly contribute to the qualitative explanation of previously described statistical non-invariance of instruments that are typically used for their assessment in Germany and China (e.g., Bieda et al., 2017; Lin et al., 2019). In addition,, the content of some items seems to no longer be relevant for the assessment of the constructs, specifically in the group of young adults in both countries. The results suggested that non-invariance partly comes from translation bias (which may cause understanding bias) as well as true cultural differences in the conception, or even the experience, of the investigated constructs. Based on our findings, we provide suggestions for how to modify the instruments to foster their measurement invariance and to improve their validity in cross-cultural investigations.

\subsection{Happiness}

The present results revealed that in both countries, happinness was, inter alia, associated with positive emotions and social interaction. The German focus groups emphasized that the term "Glück" (the verbatim translation of "happiness") implies an uncontrollable random component (e.g., luck). In contrast, the Chinese wording “幸福xìngfú (the verbatim translation of "happiness")" was reported to be typically understood as the fullfillment of a dream or the achievement of a goal.

This translation bias can influence the way individuals from Germany and China understand the items of the SHS (Item 3 and Item 4). Furthermore, based on current results, the lack of measurement invariance (Bieda et al., 2017) can also be partly explained by the complex formulation of both items. A reformulation of their wording might improve the measurement invariance and the fit of the SHS for cross-cultural investigations. Specifically, in Item 3, the phrase "getting the most out of everything" could be deleted, as well as the phrase "Although they are not depressed" in Item 4. Both were considered as especially critical and unfitting for the definition of happiness. 


\subsection{Life Satisfaction}

A common instrument to assess life satisfaction is the five-item SWLS (Diener et al., 1985). The current findings revealed that the understanding of this construct is largely similar in Germany and in China. In both countries, the level of life satisfaction was described as closely linked to different standards - to a greater extent in Germany than in China. Moreover, in both countries, quality of life is an important facet of life satisfaction. Interestingly, in the German focus groups, quality of life was linked to the satisfaction of basic needs. In contrast, the Chinese focus groups emphasized more complex needs for ecomic wealth. Overall, in both countries, the fit of the SWLS items to the understanding of life satisfaction was rated just as high. In comparison, Item 1 - a critical item in Bieda et al. (2017) - was the item with the lowest fit. In Germany and in China, the term "ideal" was criticised as something that is unnecessary to experience life satisfaction, and may even evoke negative emotions because of its inaccessibility. Therefore, it can be assumed that replacing it with a term that is more achievable (e.g., "In most ways my life is close to what I always wanted to achieve in my life/considered as important in my life") could enhance the suitability of the SWLS for cross-cultural studies.

\subsection{Perceived Social Support}

Perceived social support is often assessed by the 14 items of the German-developed F-SozU K-14 (Fydrich et al., 2009). In the present study, it was described as closely linked to interpersonal relationships in Germany and in China. However, due to a translation bias with the term "social support" in the Chinese language, the understanding of the main context and, therefore, the form of support differs between the countries (i.e., close social network in Germany vs. "the society" in China). Earlier research emphasized that Western individualistic countries focus on instrumental support, while emotional support is of greater importance in Asian collectivistic countries (e.g., Taylor et al., 2014). In contrast, in the present focus groups, emotional support was an important issue in both countries. Moreover, the fit of Item 5-that directly focuses on the relevance of instrumental support (i.e., to borrow something) for the understanding of social support-was evaluated as low in the German focus groups. In Chinese groups, it was emphasized that borrowing something from someone can cause unpleasant consequences and evoke negative emotions in their culture. Thus, it seems that the content of Item 5 - one of the items that previously lacked measurement invariance (Bieda et al., 2017; Lin et al., 2019) - is no longer up-to-date for the assessment of social support, at least for young adults in Germany and in China. Its exclusion might improve the validity of the F-SozU K-14 in cross-cultural investigations. Additionally, Item 8 might be excluded. Its content - a further item lacking measurement invariance (Bieda et al., 2017; Lin et al., 2019) - referring to the possibility of "being hugged" caused irritation in the focus groups in both countries. It implies physical contact that may be linked to individual suspensions and discomfort. This item seems to be less linked to the current understanding of social support, given that is associated with positive emotions in Germany and in China. The translation bias in the Chinese language might at least partly explain the previously reported lack of measurement invariance of Item 11 (Bieda et al., 2017; Lin et al., 2019). Its reformulation can at least partly solve this problem.

Despite its innovative character, the present study has limitations that are important to mention. First, the mostly young, female, and well-educated composition of the focus groups in both countries limits the representativity of our current findings. Due to the 
limited resources and the anonymous nature of the quantitative data, the sample selection of the current study was not based on a systematic approach but on participant willingness and random collection (in the German sample). Moreover, the participants from the two countries varied on some demographic aspects such as age range, although it reflects the true age range of university students in China and Germany. Therefore, we cannot rule out that different opinions observed from the two country groups are at least partly due to the varied age rather than the specific culture of the samples. Therefore, future studies should replicate the present study by working with more gender- and education-balanced focus groups. Considering that the understanding of the investigated constructs and their assessment may also differ across age and life experiences, more age-balanced focus groups could give further insight that is not limited to the perspective of only one cohort. Second, the size of a single focus group was rather small, and the three constructs were not discussed together in all groups, which may limit the variety of results, especially for the discussion of life satisfaction and happiness. The small group size enabled deeper and longer discussions of specific issues. However, adding participants to each focus group might bring new points of view to the discussion and, therefore, extend current findings. Third, the focus groups were conducted separately in Germany and in China without representatives from the other country/culture. Thus, there was no possibility for cross-cultural exchange during the discussions. This allowed for gathering findings that are specific to both countries. Nevertheless, to get a broader cross-cultural view of the investigated constructs, future studies should include a further type of focus groups consisting of representatives from both countries/cultures to allow for a direct exchange and, therefore, a direct evaluation of potential differences and commonalities. Note that despite their expertise and exchange, the possibility cannot be ruled out that the psychologists who moderated the focus groups and analyzed the data were at least partly biased by their own cultural background. Considering the technical progress, future studies might use a technical algorithm for some of the analysis steps to prevent "human" biases. Fourth, in the present study, German and Chinese perspectives on the investigated constructs and their measurement were assessed. Even though Germany can be considered as a representative of Western culture and China as a representative of Asian culture, both countries have specific features that differ them from other countries that also belong to the same cultural area. Therefore, to investigate the representativeness of the current findings, future studies should include focus groups from further countries to replicate the results of the present study.

In sum, it can be concluded that the different dimensions of well-being (subjective wellbeing and social well-being) can be identified and assessed in Germany and in China. However, the transfer of instruments designed to assess dimensions in the framework of Western culture to non-Western cultures by a "simple" translation should be considered with caution. The present results demonstrate that there are some basic differences in the understanding of the dimensions of well-being based on language characteristics (e.g., meaning of "Glueck", that is happiness, in the German language, or "support from the society", that is social support, in the Chinese language). As the language of a country shapes its cultural characteristics and vice versa (Günthner, 2013; Schramm \& Wüstenhagen, 2014), language characteristics are closely linked to the culture-specific conception of different well-being dimensions. If we take our findings seriously, they might even indicate that well-being is experienced differently in China and Germany, which would make it a culturally specific subjective experience. Therefore, mixed-method approaches are a promising way forward to improve the quality of cross-cultural studies: Prior to transferring an instrument to investigate constructs such as happiness, life satisfaction and perceived social support in crosscultural studies, it is important to consider the understanding of appropriate items in the 
populations of interest. As revealed by the current findings, the combination of quantitative approaches focusing on the cross-cultural measurement invariance (e.g., Bieda et al., 2017) and qualitative approaches that include focus group discussions is an effective proceeding to achieve this aim. The first step should be the testing of measurement invariance. In the second step, items that lack measurement invariance should be discussed in focus groups. This can improve the quality of cross-cultural studies and our understanding of cross-cultural differences and commonalities.

Supplementary Information The online version contains supplementary material available at https://doi. org/10.1007/s10902-021-00454-6.

Acknowledgements This study was supported by Alexander von Humboldt Professorship awarded to the last author by the Alexander von Humboldt-Foundation. The authors would like to acknowledge Valeriia Marco and Lina Neuhoff, as well as Wiebke Bruns, Christian Burk, Gwenda Kleine, Lea Knaps, Eva Lottermoser, Amelie Niemeyer, and Stephanie Schulz for their support in the data collection and management, Dr. Xiao Chi Zhang for her support in idea discussion, and Xu Wen in his support in the manuscript revision, and Rebekkah Balwin for her supports in proofreading.

Funding Open Access funding enabled and organized by Projekt DEAL. This study was supported by Alexander von Humboldt Professorship awarded to the last author by the Alexander von Humboldt-Foundation.

Availability of data and material The anonymous transcripts can be obtained by asking the correspondent author.

\section{Declarations}

Conflicts of interest The authors have no conflicts of interest to declare that are relevant to the content of this article.

Ethics approval The Ethics Committee at Ruhr Universität Bochum approved the study implementation.

Consent to participate Participants all give written informed consent before taking part in the study.

Consent for publication The manuscript is original, it has not been published previously and it is not under consideration for publication elsewhere. All authors listed have approved the publication of the manuscript. If accepted, the manuscript will not be published elsewhere.

Open Access This article is licensed under a Creative Commons Attribution 4.0 International License, which permits use, sharing, adaptation, distribution and reproduction in any medium or format, as long as you give appropriate credit to the original author(s) and the source, provide a link to the Creative Commons licence, and indicate if changes were made. The images or other third party material in this article are included in the article's Creative Commons licence, unless indicated otherwise in a credit line to the material. If material is not included in the article's Creative Commons licence and your intended use is not permitted by statutory regulation or exceeds the permitted use, you will need to obtain permission directly from the copyright holder. To view a copy of this licence, visit http://creativecommons.org/licenses/by/4.0/.

\section{References}

Berry, J. W. (1989). Introduction to methodology. In H. Triandis \& J. W. Berry (Eds.), Handbook of crosscultural psychology (Vol. 2, pp. 1-28). Allyn \& Bacon.

Bieda, A., Hirschfeld, G., Schönfeld, P., Brailovskaia, J., Zhang, X. C., \& Margraf, J. (2017). Universal happiness? Cross-cultural measurement invariance of scales assessing positive mental health. Psychological Assessment, 29(4), 408-421. https://doi.org/10.1037/pas0000353

Byrne, B. M., Shavelson, R. J., Muthén, B. (1989). Testing for the equivalence of factor covariance and mean structures the issue of partial measurement.pdf. Psychological Bulletin. 105(3) 456-466 
Chen, F. F. (2008). What happens if we compare chopsticks with forks? The impact of making inappropriate comparisons in cross-cultural research. Journal of Personality and Social Psychology, 95(5), 1005-1018. https://doi.org/10.1037/a0013193

Creswell, J. W., Plano Clark, V. L. (2017). Designing and conducting mixed methods research (3 ed.). Sage.

Diener, E. (1984). Happiness is everything, or is it? Explorations on the meaning of psychological wellbeing. Psychological Bulletin, 95(3), 542-575.

Diener, E. (2009). Subjective well-being. The Science of Well-Being, 11-58

Diener, E. D., Emmons, R. A., Larsen, R. J., \& Griffin, S. (1985). The satisfaction with life scale. Journal of Personality Assessment, 49(1), 71-75.

Diener, E., Suh, E. M., Lucas, R. E., \& Smith, H. L. (1999). Subjective well-being: Three decades of progress. Psychological Bulletin, 125(2), 276-302. https://doi.org/10.1037/0033-2909.125.2.276

Fydrich, T., Sommer, G., Tydecks, S., \& Brähler, E. (2009). Fragebogen zur sozialen Unterstützung (F-SozU): Normierung der Kurzform (K-14). Zeitschrift Für Medizinische Psychologie, 18, 43-48.

Günthner, S. (2013). Sprache und Kultur. In P. Auer, Sprachwissenschaft (pp. 347-376). JB Metzler

Helliwell, J. F., Huang, H., \& Wang, S. (2017). The social foundations of world happiness. World Happiness Report, 8.

Hines, A. M. (1993). Linking qualitative and quantitative methods in cross-cultural survey research: techniques from cognitive science. American Journal of Community Psychology, 21(6), 729-746. https:// doi.org/10.1007/BF00942245

Joshanloo, M. (2014). Eastern conceptualizations of happiness: fundamental differences with western views. Journal of Happiness Studies, 15(2), 475-493. https://doi.org/10.1007/s10902-013-9431-1

Joshanloo, M. (2019a). Investigating the relationships between subjective well-being and psychological well-being over two decades. Emotion, 19(1), 183-187. https://doi.org/10.1037/emo0000414

Joshanloo, M. (2019b). Lay conceptions of happiness: associations with reported well-being, personality traits, and materialism. Frontiers in Psychology, 10, 2377. https://doi.org/10.3389/fpsyg.2019.02377

Joshanloo, M., Bobowik, M., \& Basabe, N. (2016). Factor structure of mental well-being: contributions of exploratory structural equation modeling. Personality and Individual Differences, 102, 107-110. https://doi.org/10.1016/j.paid.2016.06.060

Joshanloo, M., Lepshokova, ZKh., Panyusheva, T., Natalia, A., Poon, W.-C., Yeung, V. W., Sundaram, S., Achoui, M., Asano, R., Igarashi, T., Tsukamoto, S., Rizwan, M., Khilji, I. A., Ferreira, M. C., Pang, J. S., Ho, L. S., Han, G., Bae, J., \& Jiang, D.-Y. (2014). Cross-cultural validation of fear of happiness scale across 14 national groups. Journal of Cross-Cultural Psychology, 45(2), 246-264. https://doi.org/ $10.1177 / 0022022113505357$

Kahneman, D., Diener, E., \& Schwarz, N. (Eds.). (1999). Well-being: Foundations of hedonic psychology. Russell Sage Foundation

Kang, S.-M., Shaver, P. R., Sue, S., Min, K.-H., \& Jing, H. (2003). Culture-specific patterns in the prediction of life satisfaction: roles of emotion, relationship quality, and self-esteem. Personality and Social Psychology Bulletin, 29(12), 1596-1608. https://doi.org/10.1177/0146167203255986

Keyes, C. L. M. (2007). Promoting and protecting mental health as flourishing: a complementary strategy for improving national mental health. American Psychologist, 62(2), 95-108. https://doi.org/10.1037/ 0003-066X.62.2.95

Keyes, C. L. (Ed.) (2012). Mental well-being: International contributions to the study of positive mental health. Springer Science \& Business Media.

Keyes, C. L. M., Shmotkin, D., \& Ryff, C. D. (2002). Optimizing well-being: the empirical encounter of two traditions. Journal of Personality and Social Psychology, 82(6), 1007-1022. https://doi.org/10.1037/ 0022-3514.82.6.1007

Kitayama, S., \& Markus, H. R. (2000). The Pursuit of Happiness and the Realization of Sympathy: Cultural Patterns of Self, Social Relations, and Well-being. In E. Diener \& E. M. Suh (Eds.), Culture and Subjective Well-Being (p. 51). MIT Press.

Krueger, R. A., \& Casey, M. A. (2015). Focus groups (5th Edition). Sage.

Kuppens, P., Realo, A., \& Diener, E. (2008). The role of positive and negative emotions in life satisfaction judgment across nations. Journal of Personality and Social Psychology, 95(1), 66-75. https://doi.org/ 10.1037/0022-3514.95.1.66

Lin, M., Hirschfeld, G., \& Margraf, J. (2019). Brief form of the Perceived social support questionnaire (F-SozU K-6): Validation, norms, and cross-cultural measurement invariance in the USA, Germany, Russia, and China. Psychological Assessment, 31(5), 609-621. https://doi.org/10.1037/pas0000686

Lu, L., \& Gilmour, R. (2004). Culture and conceptions of happiness: Individual oriented and social oriented SWB. Journal of Happiness Studies, 5(3), 269-291.

Lu, L., \& Shih, J. B. (1997). Sources of happiness: a qualitative approach. The Journal of Social Psychology, 137(2), 181-187. https://doi.org/10.1080/00224549709595429 
Lyubomirsky, S., \& Lepper, H. S. (1999). A measure of subjective happiness: preliminary reliability and construct validation. Journal Indicators Research, 46(2), 137-155.

Markus, H. R., \& Kitayama, S. (1991). Culture and the self: Implications for cognition, emotion, and motivation. Psychological Review, 98(2), 224-253.

Mayring, P. (2015). Qualitative Inhaltsanalyse: Grundlagen und Techniken (12., überarbeitete Auflage). Beltz Verlag.

McDowell, I. (2006). Measuring health: a guide to rating scales and questionnaires. Oxford University Press.

OECD (2021, February 10). Better Life Index (life satisfaction), Paris: OECD. http://www.oecdbetterlifei ndex.org/topics/life-satisfaction/.

Ryan, R. M., \& Deci, E. L. (2001). On happiness and human potentials: A review of research on hedonic and eudaimonic well-being. Annual Review of Psychology, 52(1), 141-166.

Ryff, C. D. (1989). Happiness Is Everything, or Is It? explorations on the meaning of psychological wellbeing. Journal of Personality and Social Psychology, 57(6), 1069-1081.

Scholten, S., Velten, J., Bieda, A., Zhang, X. C., \& Margraf, J. (2017). Testing measurement invariance of the depression, anxiety, and stress scales (DASS-21) across four countries. Psychological Assessment, 29(11), 1376-1390. https://doi.org/10.1037/pas0000440

Schramm, S., \& Wüstenhagen, C. (2014). Das Alphabet des Denkens: wie Sprache unsere Gedanken und Gefühle prägt. Rowohlt Verlag $\mathrm{GmbH}$.

Schreier, M. (2012). Qualitative content analysis in practice. SAGE

Shin, D. C., \& Johnson, D. M. (1978). Avowed happiness as an overall assessment of the quality of life. Social Indicators Research, 5(1-4), 475-492. https://doi.org/10.1007/BF00352944

Steenkamp, J.-B.E.M., \& Baumgartner, H. (1998). Assessing measurement invariance in cross-national consumer research.pdf. Journal of Consumer Research, 25, 78-90.

Taylor, S. E., Sherman, D. K., Kim, H. S., Jarcho, J., Takagi, K., \& Dunagan, M. S. (2004). Culture and social support: who seeks it and why? Journal of Personality and Social Psychology, 87(3), 354-362. https://doi.org/10.1037/0022-3514.87.3.354

Teismann, T., \& Brailovskaia, J. (2020). Entrapment, positive psychological functioning and suicide ideation: a moderation analysis. Clinical Psychology \& Psychotherapy, 27(1), 34-41.

The World Bank (2020, August 26). GDP per capita, PPP (current international \$) - China, Germany. https://data.worldbank.org/indicator/NY.GDP.PCAP.PP.CD?locations=CN-DE

Tov, W., \& Au, E. W. M. (2013). Comparing well-being across nations: conceptual and empirical issues. Oxford University Press. https://doi.org/10.1093/oxfordhb/9780199557257.013.0035

Vandenberg, R. J., \& Lance, C. E. (2000). A review and synthesis of the measurement invariance literature: suggestions, practices, and recommendations for organizational research. Organizational Research Methods, 3(1), 4-70. https://doi.org/10.1177/109442810031002

World Health Organization. (1948). World Health Organization Constitution. Basic Documents, 1, 22.

Publisher's Note Springer Nature remains neutral with regard to jurisdictional claims in published maps and institutional affiliations. 